NASA Technical Memorandum 102526

\title{
Performance and Modeling of Superconducting Ring Resonators at Millimeter-Wave Frequencies
}

K.B. Bhasin

National Aeronatuics and Space Administration_.

Lewis Research Center

Cleveland, Ohio

C.M. Chorey

Sverdrup Technology, Inc.

NASA Lewis Research Center Group

Cleveland, Ohio

J.D. Warner, R.R. Romanofsky, and V.O. Heinen

National Aeronautics and Space Administration

Lewis Research Center

Cleveland, Ohio

K.S. Kong, H.Y. Lee, and T. Itoh

The University of Texas at Austin

Austin, Texas

Prepared for the

1990 IEEE MTT-S International Microwave Symposium

Dallas, Texas, May 8-10, 1990

\section{N/Sh}

(NASA-TM-102526) PERFORMANCE AND MODELING OF SUPERCONDUCTING RING RESONATORS AT

MILLIMETER-WAVE FREOUENCIES (NASA) $9 \mathrm{p}$ CSCL $09 A$

N90-18634 


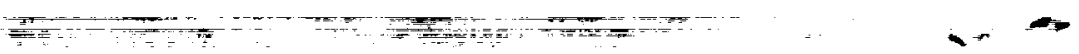




\title{
PERFORMANCE AND MODELING OF SUPERCONDUCTING RING RESONATORS AT MILLIMETER-WAVE FREQUENCIES .
}

\author{
K.B. Bhasin \\ National Aeronautics and Space Administration \\ Lewis Research Center \\ Cleveland, Ohio 44135 \\ C.M. Chorey \\ Sverdrup Technology, Inc. \\ Lewis Research Center Group \\ Cleveland, Ohio 44135
J.D. Warner, R.R. Romanofsky, and V.0. Heinen
National Aeronautics and Space Administration
Lewis Research Center
Cleveland, Ohio 44135 \\ K.S. Kong, H.Y. Lee, and T. Itoh \\ Department of Electrical and Computer Engineering \\ The University of Texas at Austin \\ Austin, TX 78712
}

\begin{abstract}
Microstrip ring resonators operating at $35 \mathrm{GHz}$ have been fabricated from laser ablated YBCO thin films deposited on lanthanum aluminate substrates. They were measured over a range of temperatures and their performance compared to identical resonators made of evaporated gold. Below $60^{\circ}$ Kelvin the superconducting strip performed better than the gold, reaching an unloaded ' $Q$ ' $\sim 1.5$ times that of gold at $25^{\circ} \mathrm{K}$. A shift in the resonant frequency follows the form predicted by the London equations. The Phenomenological Loss Equivalence Method is applied to the ring resonator and the theoretically calculated $Q$ values are compared to the experimental results.
\end{abstract}

\section{INTRODUCTION}

Recent observations of low surface resistance at microwave and millimeter wave frequencies in thin superconducting films [1] suggest their use for low loss/high $Q$ microstrip circuits. of interest is the surface resistance exhibited by these films across a wide frequency range. To date, measurements of surface resistance in the $\mathrm{Ka}$ band and above have been by the cavity technique. This technique fails to model microstrip losses completely because it neglects substrate losses and fails to adequately probe the filmsubstrate interface. Microstrip resonators patterned from thin films on microwave substrates allow direct measurement of microstrip losses. Several groups have made such measurements at lower microwave frequencies. $[2,3,4]$ In this paper we report on the direct measurement of losses by Ka band microstrip resonators made from laser ablated YBCO films on lanthanum aluminate. Also, we calculate the $Q$ values of the structure using the Phenomenological Loss Equivalence Method and invoking superposition of the internal impedances of the strip and ground plane of the microstrip line. The calculated $Q$ value of the ring resonator with a superconducting strip and a normal conducting ground plane is compared with the experimental results. 


\section{GROWTH AND PATTERNING}

The superconducting films were deposited by laser ablation of a sintered YBCO pellet onto a heated $\left(700^{\circ} \mathrm{C}\right)$ lanthanum aluminate substrate in a 100 mtorr oxygen atmosphere and then slowly cooled to room temperature in 1 atmosphere of oxygen. [5] Films with very smooth surfaces and TC's of 89.8 have been produced; X-ray analysis has shown that they are c-axis aligned. The microstrip resonators were patterned by standard photolithography using negative photoresist and a 'wet' chemical etchant. This etchant was either a 38 solution of bromine in ethanol or dilute phosphoric acid in water. A metal ground plane was deposited by first evaporating 100 $\AA$ of $T i$ for adhesion followed by 1 micron of gold. In addition to the resonator, each chip also had a test bar for direct Tc testing of the patterned film. Identical resonators were fabricated entirely from gold (both strip and ground plane) using evaporation and lift-off to define the strip.

The resonators were measured using a Hewlett-Packard 8510 Network Analyzer, operating in WR-28 waveguide. The microstrip circuit was mounted in a tapered ridge waveguide to microstrip test fixture which was mounted at the second stage of a two stage, closed cycle helium refrigerator. Circuit temperatures reached approximately $20^{\circ} \mathrm{K}$ and were monitored by a silicon diode sensor mounted in the test fixture. The entire cold finger and test fixture were enclosed in a custom designed vacuum can. Microwave coupling to the test fixture was through 6 inch sections of WR-28 waveguide made of thin wall stainless steel to minimize heat conduction. Vacuum was maintained at the waveguide feedthroughs by means of ' $O$ ' rings and mica sealing windows.

\section{THEORETICAL CALCULATION OF Q}

The theoretical $Q$ values were calculated using the Phenomenological Loss Equivalence Method (PEM). [6] This method is applicable to cases where the strip conductor thickness is on the order of a skin depth (for a normal metal) or a penetration depth (for a superconductor). The Incremental Inductance Rule, which is often used to calculate microstrip losses, can only be applied in the case of shallow field penetration, which is not satisfied in this study. Also, PEM has the advantage of simple calculation compared with other numerical techniques such as the Finite Element Method. The technique proceeds on the basis of separately calculating the internal impedances of the strip and the ground plane through use of an equivalent isolated strip, and then adding these impedances to the external impedance of the microstrip structure. First, the ground plane is assumed to be a perfect conductor so that there is no magnetic field penetration into it as shown in figure 1. A geometric factor (G1) for the strip line is then obtained from the magnetic field penetration into it. This G1 factor is used to obtain an equivalent strip; from which the internal impedance of the microstrip line under the assumption of perfect ground plane can be obtained as

$$
\mathrm{Z}_{\mathrm{i} 1}=\mathrm{G}_{1} \cdot \mathrm{Z}_{\mathrm{s} 1} \cdot \operatorname{coth}\left(\mathrm{Z}_{\mathrm{s} 1} \bullet \sigma_{1} \cdot \mathrm{A} \cdot \mathrm{G}_{1}\right)
$$




\section{ORIGINAL PAGE IS \\ OF POOR QUALITY}

where $\mathrm{Z}_{11}, \sigma_{1}$ and $\mathrm{A}$ are the surface impedance, the conductivity of the material and the cross sectional area of the strip, respectively. Next we consider the strip as a perfect conductor as shown in figure 1. Then a geometric factor (G2) is obtained for the field penetration into the ground plane. With the value G2, we obtain the internal impedance of the ground plane based on the assumption of a perfect strip,

$$
\mathrm{Z}_{\mathrm{i} 2}=\mathrm{G}_{2} \cdot \mathrm{Z}_{\mathrm{s} 2} \cdot \operatorname{coth}\left(\mathrm{Z}_{\mathrm{s} 2} \cdot \sigma_{2} \cdot \mathrm{A} \cdot \mathrm{G}_{2}\right)
$$

where $\mathrm{Z}_{\mathrm{sz}}$ and $\sigma_{2}$ are surface impedance and conductivity of the ground, respectively. The internal impedance of the microstrip line is obtained by adding $\mathrm{Z}_{11}$ and $\mathrm{z}_{12}$. We add this internal impedance to the externa 1 inductance and calculate the propagation constant of the microstrip line by using a

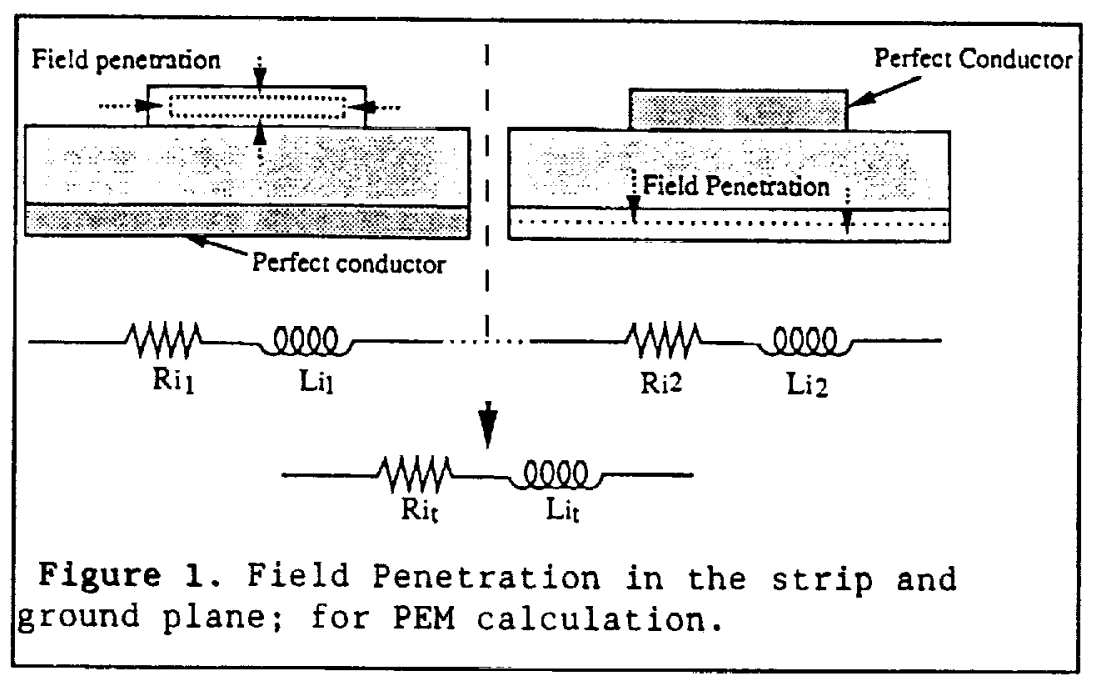
transmission line

model. It should be emphasized that (1) and (2) are applicable to any field penetration depth.

The conductor losses of the structure in fig. 2 were calculated by applying the method explained above. Then, the $Q$ values of each resonator were calculated by additional consideration of substrate

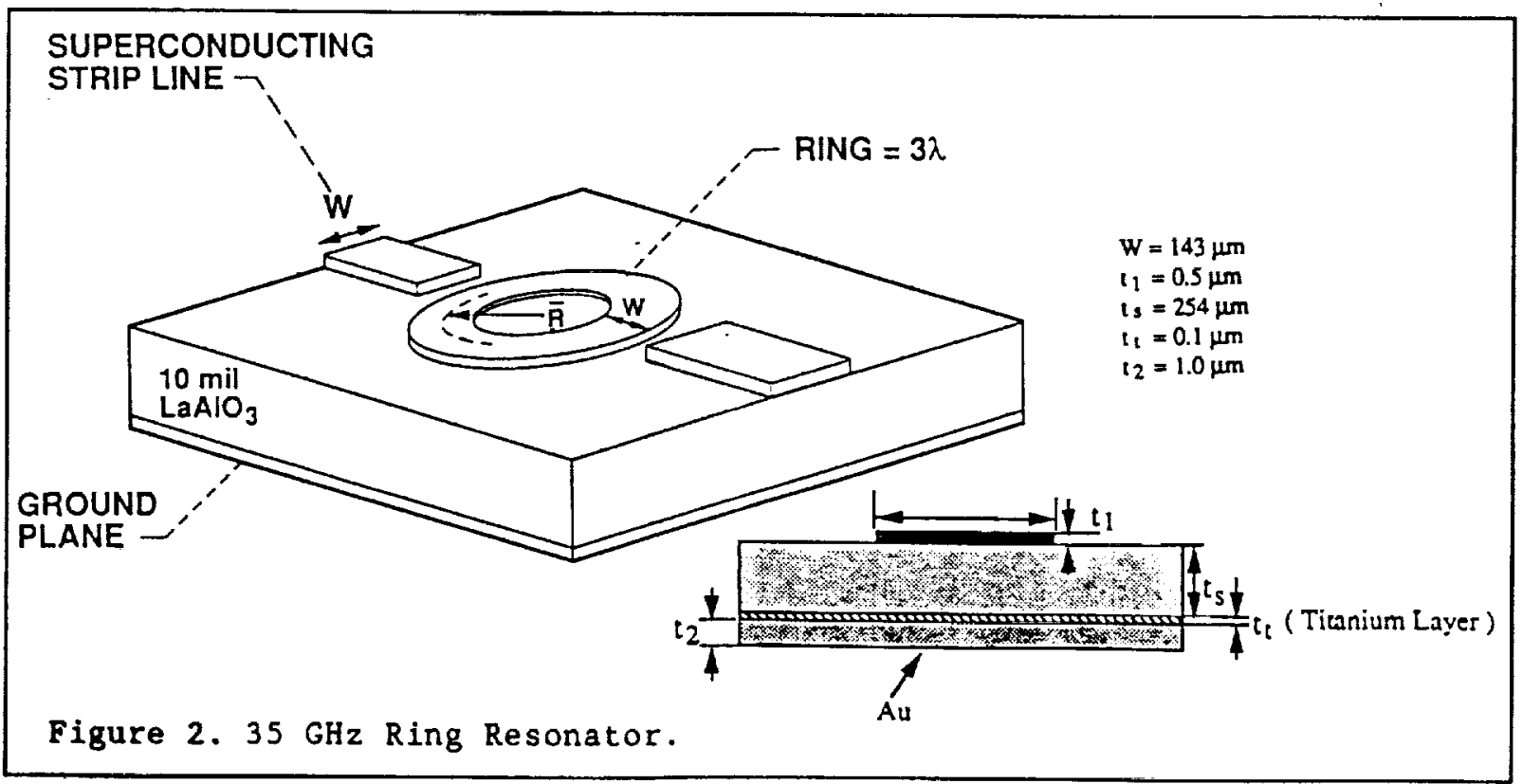


loss; radiation loss wa $s$ a $s$ u m e d negligible. For the calculation, the value of $5.8 \times 10-4$ was used for the loss tangent. Since the current is more concentrated on the $s t r i p, t h e$ implementation of a superconductor in the strip has more influence on the loss. The extent of the effect of implementing a superconductor in the microstrip line can be different for different geometries.

RESULTS AND DISCUSSION

In figure 3 are shown plots of Sil for a superconducting resonator at several

temperatures. This plot is of the reflected power from the resonator in the test fixture and is thus a measure of the loaded ' $Q$ '. Two features are apparent; 1) the coupling changes with temperature (in this case, starting at near critical coupling just below Tc and going to overcoupled at lower temperatures), and 2) the resonant frequency shifts with temperature. The change in the resonant frequency vs temperature is plotted in figure 4 along with the resonant frequencies of a gold resonator. The variation observed in the gold resonator follows the form expected from thermal contraction in the substrate. But since accurate data on lanthanum aluminate is not readily available, frecise comparisons are not possible. The variation seen in tne superconducting resonator is a consequence of the dependence of the internal impedance of the strip on the changing normal/superconducting electron densities. The internal inductance of a superconducting strip over a ground plane is given by:[7]

$$
L_{1 n t}=\mu_{0} \cdot \lambda \cdot \operatorname{coth}(t / \lambda)
$$

Assuming the Gorter-Casimir temperature dependence of $\lambda$ :

$$
\lambda(\mathrm{T})=\frac{\lambda_{\mathrm{o}}}{\left[1-(\mathrm{T} / \mathrm{TC})^{4}\right]^{4}}
$$

the form of the resonant frequency variation based on the changing 


\section{ORIGINAL PAGE IS \\ OF POQR QUALITY}

line inductance matches the experimental observations. However, attempts at numerical fitting to extract $\lambda_{0}$, result in $\lambda_{0}$ in excess of 1 micron, indicating that the film quality may not be at its highest.

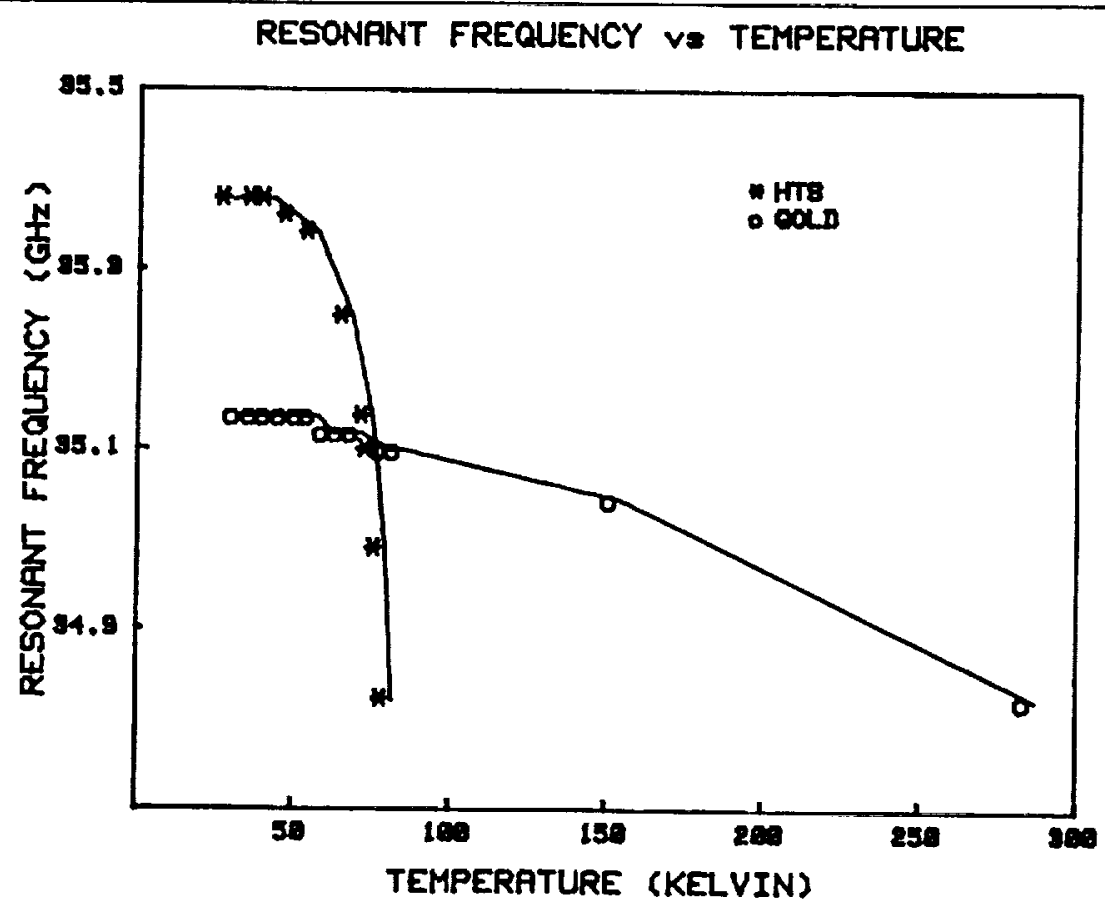

Figure 4. Resonant frequencies vs temperature for superconducting and normal strips.

The best circuit to date has been from a $6500 \AA$ film with a postprocessing TC of $79^{\circ} \mathrm{K}$. The unloaded $Q$ of this circuit is plotted against temperature in figure 5 along with the unloaded $Q$ of an identical gold resonator. The $Q$ of the superconducting circuit rises sharply below Tc, exceeding the $Q$ of the gold circuit at $\sim 60^{\circ} \mathrm{K}$ and reaching a value of 1.5 times that of the gold resonator at $25^{\circ} \mathrm{K}$. Comparing the experimental results with the calculated values in the same figure, we see that for the gold resonator, the PEM calculation matches the experimental fairly well. The measured superconducting ' $Q$ ', however, is much lower than the calculated values. Several reasons can be given for this. First, the values for the complex conductivity of the superconductor used in the PEM calculation were obtained by microwave reflectance/transmission measurements on separate laser ablated films. [8] It is likely that the quality of those films was higher than the resonator film, in part because these films were unpatterned. In addition, substrate losses in the PEM were calculated on the basis of $\tan \delta=5.8 \times 10 \mathrm{E}-4$ but accurate values for lanthanum aluminate are not available so the actual value may be higher or lower. It seems likely that improvements in the measured $Q$ are possible with increased film quality. 


\section{CONCLUSIONS}

Ring resonator circuits were fabricated from laser ablated YBCO superconducting films on lanthanum aluminate to determine transmission line losses at millimeter wave frequencies. At $25^{\circ} \mathrm{K}$ the unloaded $Q$ of the superconducting resonator was 1.5 times the $Q$ of identical resonators made of gold. A shift in the resonant frequency witli temperature follows the form predicted by the London equation. Using the PEM we calculated the $Q$ values of the ring resonator with a thin YBCO strip and a gold ground plane. The theoretical results were compared with experimental results of the ring resonator of that structure. The calculated results predict higher values of $Q$ than those actually observed, but improved film quality should increase measured $Q$ values.

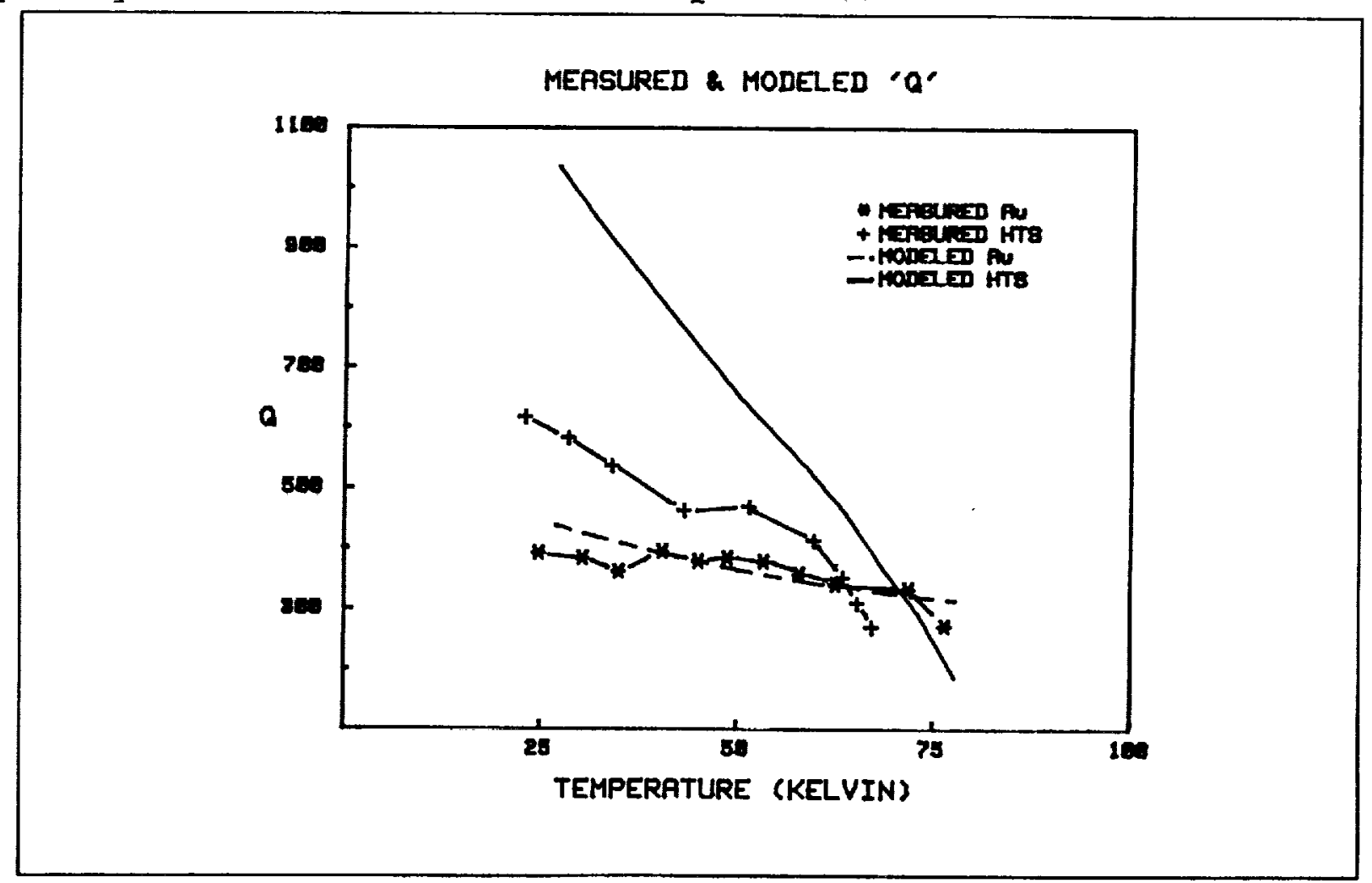

Figure 5. Measured and calculated values of unloaded $Q$ for superconducting and normal resonators. 


\section{REFERENCES}

1. N. Klein, G. Muller, H. Piel, B. Roas, L. Schultz, U. Klein and M. Peiniger, "Millimeter Wave Surface Resistance of Epitaxially Grown YBCO Thin Films," Appl. Phys. Lett. Vol. 54, pp 757-759.

2. A. A. Valenzuela and P. Russer, "High Q Coplanar Transmission Line Resonators of YBCO on MgO," Appl. Phys. Lett., Vol. 55, pp. 1029-1031, 1989.

3. B. R. McAroy, G. R. Wagner, J. D. Adam, J. Talvacchio and M. Driscoll, "Superconducting Stripline Resonator Performance," IEEE Trans. Magn., Mag. Vol. 25, pp. 1104-1106 (1989).

4. J. H. Takemoto, F. K. Oshita, H. R. Fetterman, P. Kobrin, and E. Sovoro, "Microstrip Ring Resonator Technique for Measuring Microwave Attenuation in High-TC Superconducting Thin Films," IEEE Trans. Microwave Theory and Tech., Vol. MTT-37, pp. 1650-1652, 1989 .

5. J. D. Warner, K. B. Bhasin, N. C. Varaljay, D. Y. Bohman and C. M. Chorey, "Growth and Patterning of Laser Ablat $ə$ d Superconducting YBCO Films on LaAlO3 Substrates," NASA TM-102336.

6. H. Y. Lee, and T. Itoh, "Phenomenological Loss Equivalence Method for Planar Quasi-TEM Transmission Line with a Thin Normal Conductor or Superconductor," IEEE Trans. Microwave Theory and Tech., Vol. MTT-37, no. 12, pp. 1904-1909, 1989.

7. James Swihart, "Field Solution for a Thin-Film Superconducting Strip Transmission Line," Journal Appl. Phys., Vol 32, no. 3, pp. 461-469, 1961 .

8. F. A. Miranda, W. L. Gordon, K. B. Bhasin, V. O. Heinen, and J. Valco, "Millimeter Wave Transmission Studies of YBCO Thin Films in the 26.5 to $40 \mathrm{GHz}$ Frequency Range," Proc. Third Annual Conf. on Superconductivity and Applications, to be published by Plenum Press 1990, and NASA TM-102345. 


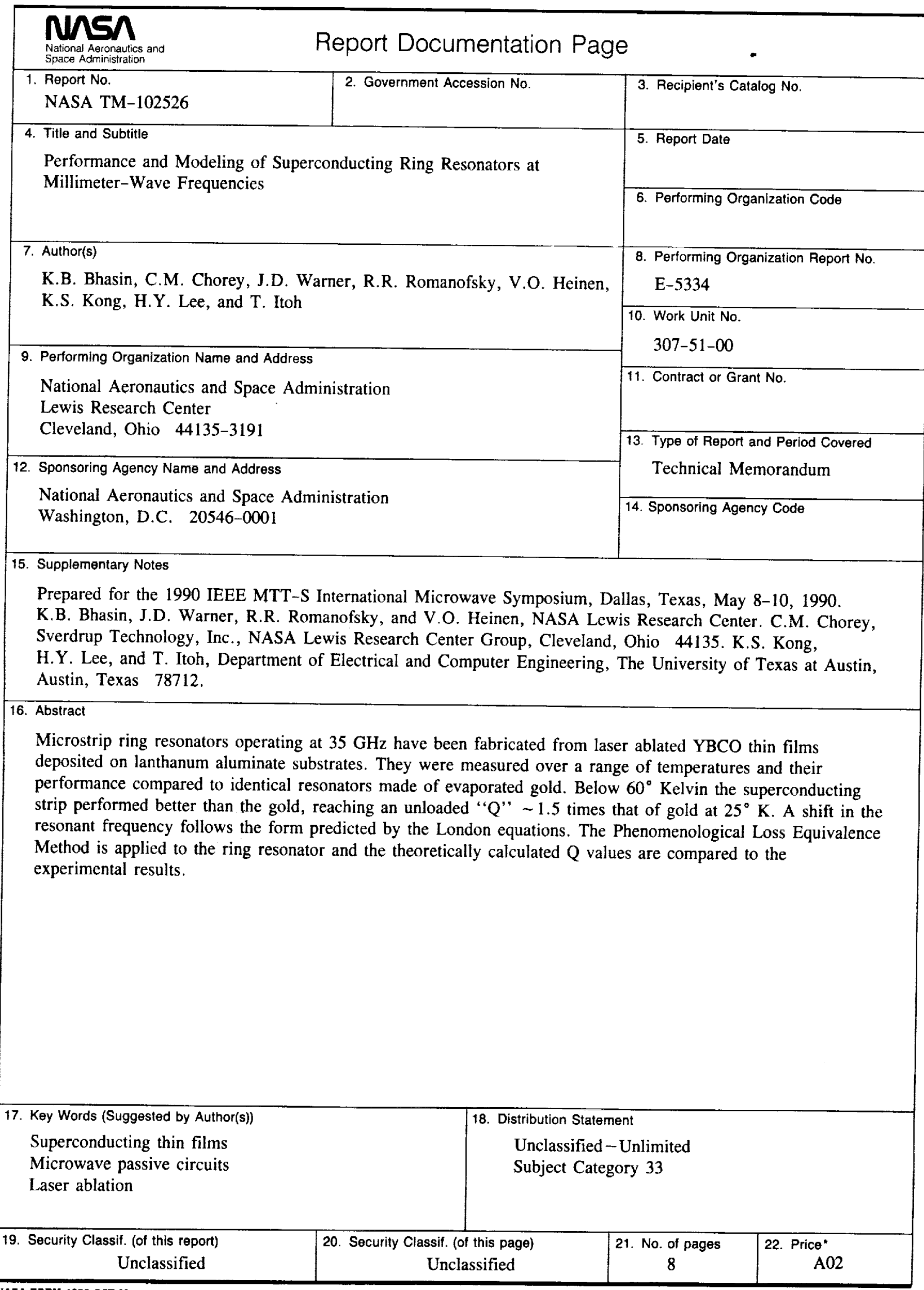

